\title{
O papel da produção de conhecimento tecnológico na internacionalização das empresas industriais brasileiras
}

Resumo: O objetivo deste trabalho é estimar o efeito da produção de conhecimento tecnológico das empresas industriais brasileiras sobre suas respectivas probabilidades condicionais de internacionalização, via exportações. Um estudo empírico utilizando (i) microdados da Pesquisa de Clima de Investimentos no Brasil (Banco Mundial) e (ii) técnicas de regressão não linear com variáveis instrumentais é realizado com esta finalidade. Os resultados encontrados sugerem que a probabilidade condicional de ingresso no mercado externo pode aumentar, no mínimo, em 23 pontos percentuais.

Palavras-chave: Inovação; Internacionalização; Probit; Variáveis instrumentais.

Classificação JEL: C25; F10; O31.

\footnotetext{
"Doutor em Economia pela Università degli Studi di Siena. Professor Adjunto do Departamento de Economia da Universidade Federal do Paraná (UFPR). Endereço eletrônico: esteves@ufpr.br.
} 


\section{Introdução}

O objetivo deste trabalho é estimar o efeito da produção de conhecimento tecnológico das empresas industriais brasileiras (inovações de produto e de processo) sobre suas respectivas probabilidades condicionais de internacionalização (exportações diretas). Um estudo empírico utilizando os microdados de empresas amostradas pela Pesquisa de Clima de Investimentos no Brasil, 2003 (divulgada pelo Banco Mundial) é desenvolvido com tal finalidade.

O esforço de produção de conhecimento tecnológico das empresas é mensurado através de uma variável multinomial reportando se cada uma das empresas que constitui a amostra obteve alguma inovação de produto, inovação de processo ou ambas. O grau de internacionalização das empresas é mensurado através de uma variável categórica binária (dummy), informando se cada uma das empresas amostradas é exportadora direta ou não.

É bem conhecido na literatura o fato da relação entre inovação e internacionalização apresentar bicausalidade. Um teste empírico de tal relação exige cautela, pois, nos casos onde a bicausalidade está presente, a hipótese de exogeneidade dos regressores é demasiadamente restritiva. Com o objetivo de se evitar a obtenção de estimativas enviesadas e inconsistentes para a relação populacional entre inovação e internacionalização, o presente trabalho utiliza estimadores de variáveis instrumentais.

Ainda no que diz respeito à estratégia de identificação do esforço de produção de inovação sobre a internacionalização, cabe mencionar que a variável dependente do modelo econométrico é a variável internacionalização, ou seja, uma variável categórica para determinar se a empresa é exportadora direta ou não. Dadas (i) a não linearidade da variável internacionalização e (ii) a simultaneidade da relação inovação-internacionalização, a identificação do efeito desejado será obtida através de um modelo Probit com variáveis instrumentais (aqui denominado IV-PROBIT).

Ao longo deste trabalho o autor apresenta resposta para a seguinte pergunta: qual o impacto (em pontos percentuais) que o desenvolvimento de uma inovação específica exerce sobre a probabilidade condicional de uma empresa industrial tornar-se exportadora? Dada a estrutura do modelo econométrico, algumas questões adicionais são respondidas a partir dos resultados obtidos nas regressões auxiliares (primeiro estágio do modelo IV-PROBIT): (i) qual o impacto de um determinado montante de gastos em pesquisa de desenvolvimento (P\&D) sobre a capacidade de produção de inovações? (ii) qual o impacto da cooperação universidade-empresa sobre a produção de conhecimento tecnológico?

No que diz respeito à motivação do trabalho, cabe mencionar que as perguntas aqui endereçadas são relevantes para o debate em torno do desenho 
e da avaliação de políticas públicas de promoção de exportações, tendo assim implicações tanto micro quanto macroeconômicas. O ingresso e a permanência de um número cada vez maior de empresas no setor exportador são de fundamental importância tanto para a sobrevivência e crescimento das firmas (DeNegri et al., 2007), quanto para o equilíbrio das contas externas do país (Markwald; Puga, 2002).

O presente artigo é composto por cinco seções, incluindo a presente introdução. Na segunda seção é apresentado o referencial teórico; a terceira seção é dedicada para a apresentação dos dados, das variáveis e suas respectivas estatísticas descritivas; a quarta seção é dedicada à apresentação do modelo econométrico, estratégia de identificação e resultados; a quinta e última seção é dedicada às considerações finais.

\section{Referencial teórico}

A análise econômica sobre os determinantes da internacionalização das firmas tem recebido grande atenção por parte de um grande conjunto de pesquisadores internacionais. Um fato estilizado da literatura é que um alto nível de produtividade constitui condição fundamental para determinar o grau de internacionalização de uma firma. Os custos de entrada incorridos pelas firmas para operarem em mercados externos exigem que as mesmas obtenham elevados ganhos de eficiência, ex ante (Melitz, 2003, Bernard; Jensen, 1999, 2004).

O trabalho seminal de Helpman e outros (2004) fornece a base teórica para a compreensão da relação entre heterogeneidade produtiva e diferentes graus de internacionalização das firmas. Os autores introduzem firmas heterogêneas dentro de um modelo multisetorial com múltiplos países e apenas as empresas mais produtivas são capazes de se engajarem em operações externas. As operações externas envolvem exportações e investimentos externos diretos (IED). Os autores demonstram ainda que dentro do grupo de empresas que operam externamente, apenas as mais produtivas são capazes de se engajarem em IED. Girma e outros (2005) sintetizam os resultados de Helpman e outros (2004) nos seguintes termos:

[...] Helpman, Melitz and Yeaple argue firm heterogeneity leads to self-selection in the structure of international commerce. Only the most productive firms find it profitable to meet the higher costs associated with FDI; the next set of firms finds it profitable to serve foreign markets through exporting; while the least productive firms serve only the domestic market. (Girma et al., 2005). 
Helpman e outros (2004) apresentam evidência empírica corroborando tal hipótese, utilizando dados de empresas norte-americanas. Wagner (2006) encontra evidências similares utilizando um painel de empresas alemãs. No caso brasileiro tal evidência também é corroborada e os resultados são fornecidos por Prochnik e outros (2006), enquanto que Greenaway e Kneller (2007) fornecem um survey da literatura empírica sobre o tema.

No entanto, tal literatura não aponta com clareza quais os fatores que determinam estes ganhos de eficiência ex ante (Esteve-Perez; Rodriguez, 2009). A forte interdependência macroeconômica entre exportações e inovação, verificada por Grossman e Helpman (1991), parece indicar uma pista sobre a dinâmica desse processo de ganhos de eficiência em termos microeconômicos: os ganhos de eficiência são obtidos a partir da maior capacidade de inovação das empresas.

A capacidade de inovação das firmas pode (i) tanto servir como determinante de ganhos de produtividade, habilitando assim as empresas a suportarem os custos de entrada do processo de internacionalização (Araújo, 2006), (ii) quanto a capacidade de gerar inovação e obter maior produtividade e eficiência pode ser influenciada positivamente pelo ingresso das firmas no mercado internacional (Araújo et al., 2009).

A bicausalidade da relação inovação-internacionalização torna a estimativa do efeito da inovação sobre a internacionalização não trivial. A seção 4 deste artigo apresentará com algum grau de detalhamento a estratégia empírica a ser utilizada para tentar lidar com tal problema.

Faz-se importante mencionar que este trabalho considera apenas o fato das empresas atenderem ou não o mercado externo, via exportações diretas, como critério de internacionalização das firmas. Não há informação disponível na base de dados a respeito de IED das empresas amostradas.

Um segundo ponto a ser mencionado diz respeito a uma hipótese implícita ao longo deste trabalho: tudo o mais constante, as empresas que conseguirem alcançar elevados níveis de produtividade ex ante (níveis condizentes com a cobertura dos custos de entrada no mercado externo) exercerão a opção de fornecimento ao mercado externo. Mesmo considerando todos os benefícios da atividade exportadora para as empresas, a hipótese acima será tão restritiva quanto pior forem as condições geradas no ambiente macroeconômico (câmbio valorizado e baixo crescimento da demanda externa).

\section{Dados e estatísticas descritivas}

A base de dados utilizada neste teste empírico é o World Bank Investment Climate, Brazil (2003). Esta pesquisa fornece informações individuais de 1.642 
empresas industriais brasileiras entrevistadas no ano de 2003, porém as informações disponíveis referem-se ao exercício anterior, $2002^{1}$. O uso de pesos amostrais torna a pesquisa representativa para 17.631 empresas industriais brasileiras.

O Quadro 1 apresenta a lista de variáveis a serem utilizadas ao longo deste trabalho. Já na Tabela 1 o leitor encontrará as respectivas estatísticas descritivas para o conjunto de variáveis listadas no Quadro 1. As estatísticas descritivas são reportadas para todo o conjunto de empresas da amostra e, separadamente, para os conjuntos de empresas não exportadoras (internacionalização $=0$ ) e exportadoras (internacionalização $=1$ ).

Quadro 1 - Lista de variáveis

\begin{tabular}{|c|c|}
\hline Variável & Descrição \\
\hline Internacionalização & $\begin{array}{l}\text { Variável binária: Exportou diretamente em } 2002=1 \text {; caso } \\
\text { contrário }=0\end{array}$ \\
\hline Inova Produto & $\begin{array}{l}\text { Variável binária: Empresa inovou produto nos últimos dois } \\
\text { anos }=1 \text { (2000 e 2001), caso contrário }=0 .\end{array}$ \\
\hline Inova Processo & $\begin{array}{l}\text { Variável binária: Empresa inovou produto nos últimos dois } \\
\text { anos }=1 \text { ( } 2000 \text { e 2001), caso contrário }=0 .\end{array}$ \\
\hline Esforço Inovativo & $\begin{array}{l}\text { Variável multinomial }=0 \text { para não inovadoras; }=1 \text { para } \\
\text { inovadoras de processo }=2 \text { para inovadoras de produto } ;=3 \\
\text { para inovadoras de produto e processo. }\end{array}$ \\
\hline Gastos P\&D & $\begin{array}{l}\text { Gastos em R\$ em P\&D por trabalhador empregado } \\
\text { (trabalhador tempo integral não terceirizado). }\end{array}$ \\
\hline Interação Universidade-Empresa & $\begin{array}{l}\text { Variável binária: a interação com universidades esteve } \\
\text { entre as três principais fontes de conhecimento para } \\
\text { adquirir novas tecnologias nos últimos } 2 \text { anos }=1 \text {, caso } \\
\text { contrário }=0 \text {. }\end{array}$ \\
\hline Restrição Incentivo Importação & $\begin{array}{l}\text { Variávelbinária:Restriçãoàisenção detarifas sobreinsumos, } \\
\text { matérias-primas importadas }=1 \text {, caso contrário }=0 \text {. }\end{array}$ \\
\hline Restrição Incentivo Federal & $\begin{array}{l}\text { Variável binária: Restrição a incentivos federais ao } \\
\text { investimento }=1 \text {, caso contrário }=0 \text {. }\end{array}$ \\
\hline Restrição Incentivo Municipal & $\begin{array}{l}\text { Variável binária: Restrição a incentivos municipais ao } \\
\text { investimento }=1 \text {, caso contrário }=0 \text {. }\end{array}$ \\
\hline Escolaridade & $\begin{array}{l}\text { Escolaridade média dos empregados da empresa (em anos } \\
\text { de estudo). }\end{array}$ \\
\hline Emprego & $\begin{array}{l}\text { Número de trabalhadores em tempo integral, não } \\
\text { terceirizados. }\end{array}$ \\
\hline Idade da Empresa & Idade da empresa em anos. \\
\hline Local & Variável binária: dummies para unidades da federação. \\
\hline Setor & Variável binária: dummies para setores produtivos. \\
\hline
\end{tabular}

Fonte: Elaborado pelo próprio autor.

Tabela 1 - Estatísticas descritivas

\begin{tabular}{l|r|r|r}
\hline \multicolumn{1}{c|}{ Variável } & $\begin{array}{c}\text { Empresas } \\
\text { Todas }\end{array}$ & $\begin{array}{c}\text { Empresas } \\
\text { Internacionalização = 0 }\end{array}$ & $\begin{array}{c}\text { Empresas } \\
\text { Internacionalização = 1 }\end{array}$ \\
\hline Internacionalização & \multicolumn{4}{c|}{0,225} & 0,000 & 1,000 \\
& $(0,417)$ & $(0,000)$ & $(0,000)$ \\
Inova Produto & 0,657 & 0,635 & 0,732 \\
& $(0,474)$ & $(0,481)$ & $(0,442)$ \\
Inova Processo & 0,645 & 0,627 & 0,705 \\
Inova Produto e & $(0,478)$ & $(0,483)$ & $(0,456)$ \\
Processo & 0,459 & 0,436 & 0,539 \\
\hline
\end{tabular}

1 Há informação disponível para o triênio 2000-2002 no caso de informações contábeis e patrimoniais das firmas. 
Tabela 1 (Continuação) - Estatísticas descritivas

\begin{tabular}{|c|c|c|c|}
\hline Variável & $\begin{array}{c}\text { Empresas } \\
\text { Todas }\end{array}$ & \begin{tabular}{c|} 
Empresas \\
Internacionalização $=0$
\end{tabular} & $\begin{array}{c}\text { Empresas } \\
\text { Internacionalização }=1\end{array}$ \\
\hline Esforço Inovativo & $\begin{array}{r}1,960 \\
(1,128)\end{array}$ & $\begin{array}{r}1,898 \\
(1,145)\end{array}$ & $\begin{array}{r}2,169 \\
(1,040)\end{array}$ \\
\hline Gastos P\&D & $\begin{array}{r}878,10 \\
(4513,92)\end{array}$ & $\begin{array}{r}544,41 \\
(3171,50)\end{array}$ & $\begin{array}{r}2054,79 \\
(7415,63)\end{array}$ \\
\hline Interação & 0,017 & 0,013 & 0,031 \\
\hline Universidade-Empresa & $(0,131)$ & $(0,115)$ & $(0,174)$ \\
\hline Restrição Incentivo & 0,715 & 0,742 & 0,622 \\
\hline Importação & $(0,451)$ & $(0,437)$ & $(0,484)$ \\
\hline Restrição Incentivo & 0,735 & 0,754 & 0,668 \\
\hline Federal & $(0,441)$ & $(0,430)$ & $(0,470)$ \\
\hline Restrição Incentivo & 0,759 & 0,774 & 0,709 \\
\hline Municipal & $(0,427)$ & $(0,417)$ & $(0,454)$ \\
\hline Escolaridade & $\begin{array}{r}8,065 \\
(1,892)\end{array}$ & $\begin{array}{r}7,937 \\
(1,867)\end{array}$ & $\begin{array}{r}8,508 \\
(1,912)\end{array}$ \\
\hline Emprego & $\begin{array}{r}76,677 \\
(267,497)\end{array}$ & $\begin{array}{r}41,702 \\
(76,500)\end{array}$ & $\begin{array}{r}197,110 \\
(528,336)\end{array}$ \\
\hline Idade da Empresa & $\begin{array}{r}16,426 \\
(16,066)\end{array}$ & $\begin{array}{r}14,286 \\
(13,778)\end{array}$ & $\begin{array}{r}23,794 \\
(20,575)\end{array}$ \\
\hline Observações & 1642 & 1163 & 479 \\
\hline Observações c/peso & 17361 & 13453 & 3907 \\
\hline
\end{tabular}

Fonte: Elaborado pelo próprio autor.

Nota: Desvio-padrão entre parênteses.

A primeira coluna de resultados da Tabela 1 é dedicada à apresentação das estatísticas descritivas de todas as empresas da amostra, enquanto que a segunda e terceira coluna de resultados são as descritivas das empresas não exportadoras e exportadoras, respectivamente. Das 1.642 empresas da amostra, apenas 479 empresas exportaram diretamente seus produtos no ano de 2002 (aproximadamente 29\% de empresas exportadoras). No entanto, faz-se necessário mencionar que as descritivas são reportadas tomando em conta os pesos amostrais, deste modo temos 3.907 empresas exportadoras de um total de 17.361 empresas representadas através da utilização de pesos amostrais, ou seja, o percentual de empresas exportadoras é de aproximadamente $23 \%$.

As variáveis de inovação (Inova Produto, Inova Processo e Inova Produto e Processo) são variáveis binárias, como pode ser verificado no Quadro 1. Logo, as descritivas destas variáveis são exatamente o percentual de empresas inovadoras (por categoria) em cada grupo amostral. Pode-se verificar na Tabela 1 que o percentual de empresas inovadoras no grupo de exportadoras é sempre maior do que no grupo de não exportadoras. Este diferencial alcança 10 pontos percentuais nos casos das inovações de produto $(73,2 \%$ para exportadoras e $63,5 \%$ para não exportadoras) e produto e processo (53,9\% e 43,6\%), enquanto que no caso da inovação de processo este diferencial é de 8 pontos percentuais (70,5\% e $62,7 \%$, respectivamente).

A variável esforço inovativo é uma variável multinomial com valor igual a zero para o caso das empresas que não desenvolveram nenhuma inovação no período 2000-2002, valor igual a um para as empresas que desenvolveram apenas 
inovações de processo, valor igual a dois para as empresas que desenvolveram apenas inovações de produto e valor igual a três para as empresas que desenvolveram tanto inovações de produto quanto inovações de processo no período. Uma hipótese assumida na construção desta variável é que as inovações de produto demandam maior esforço do que as inovações de processo ${ }^{2}$.

A média da variável esforço inovativo é de 1,96 para o conjunto de todas as empresas da amostra, enquanto que para o grupo de empresas não exportadoras esta média é de aproximadamente 1,90. Já no grupo de empresas exportadoras esta média é maior, ou seja, aproximadamente 2,17 , porém o erro padrão é inferior àquele apresentado pelo grupo de empresas não exportadoras. Isto significa que o grupo de empresas exportadoras apresenta maiores níveis de esforço inovativo e são mais homogêneas neste aspecto que as empresas não exportadoras.

Quando analisamos as descritivas relativas àquelas variáveis que constituem os insumos de uma função de produção de conhecimento, verificamos que as empresas exportadoras investem 3,8 vezes mais recursos em pesquisa em desenvolvimento do que as empresas não exportadoras. Já no quesito interação com universidades para desenvolvimento e absorção de tecnologias, verificamos que o percentual de empresas engajadas nesta atividade é três vezes maior para o grupo de exportadoras quando comparado com o grupo das não exportadoras.

Constatamos ainda que o percentual de empresas que declaram sofrer alguma restrição aos diferentes incentivos dos governos é sempre elevado: entre $62 \%$ e $78 \%$. No entanto, tais percentuais são sempre inferiores para o grupo de empresas exportadoras. No caso das restrições aos incentivos da esfera federal, tal diferencial chega a ser de 8,6 pontos percentuais.

Quando analisamos os demais atributos das empresas, pode-se constatar que as exportadoras reúnem trabalhadores mais qualificados, são empresas de maior porte e mais maduras do que as empresas não exportadoras.

\section{Modelo econométrico}

\subsection{Estratégia de identificação}

Já foi mencionado na introdução deste trabalho que o objetivo do mesmo é estimar o efeito da produção de conhecimento tecnológico das empresas industriais brasileiras (inovações de produto e de processo) sobre suas respectivas probabilidades condicionais de internacionalização (exportações diretas). O

\footnotetext{
2 Esta hipótese é corroborada nos dados apresentados neste trabalho. As empresas que declararam desenvolver apenas inovações de produto despenderam o dobro dos investimentos em P\&D (gastos anuais por trabalhador) gastos pelas empresas que declararam ter apenas desenvolvido inovações de processo, aproximadamente $\mathrm{R} \$ 1.500$ e $\mathrm{R} \$ 750$, respectivamente.
} 
efeito da inovação sobre a internacionalização das firmas pode ser especificado conforme o modelo econométrico abaixo:

$$
\operatorname{pr}\left(y_{i}=1\right)=\phi\left(y_{i}, Y_{i} \beta+X_{1 i} \gamma\right)=\phi\left(y_{i}, Z_{i} \delta\right)
$$

Onde $\phi$ é função densidade acumulada, $y_{i}$ é a variável binária dependente internacionalização, com valor igual a um caso a empresa $i$ seja exportadora direta e valor igual a zero caso contrário; e $Y_{i} \beta+X_{1 i} \gamma$ é a função índice onde $Y$ é a variável multinomial intensidade de inovação com valor igual a zero para empresas não inovadoras, igual a um para empresas inovadoras de processo, igual a dois para empresas inovadoras de produto e igual a três para empresas que tenham desenvolvido tanto inovações de produto quanto inovações de processo; $X_{1 i}$ é um vetor de variáveis exógenas de controle da firma $i$. A Equação (1) implica que $Y_{t}$ e $X_{1 t}$ sejam incluídas como regressores do modelo PROBIT e a função de máxima verossimilhança (MVS) é maximizada com respeito a $\delta^{\mathrm{T}}=\left[\beta^{\mathrm{T}}, \gamma^{\mathrm{T}}\right]$.

O modelo usual MVS-PROBIT pode ser utilizado para estimar os parâmetros $\delta^{\mathrm{T}}$. No entanto, quando qualquer um dos regressores é endógeno $\left(Y_{i}\right.$, por exemplo), a estimativa de seu parâmetro $(\beta)$ será não só enviesada, como também inconsistente (Yatchew; Griliches, 1985).

Como já mencionado anteriormente, a hipótese de exogeneidade da variável $Y_{i}$ na Equação (1) é demasiadamente restritiva, pois é amplamente reconhecido na literatura a bi causalidade entreinovação $\left(Y_{i}\right)$ einternacionalização $\left(y_{i}\right)$. Essa bicausalidade impossibilita que o pesquisador obtenha parâmetros não enviesados e consistentes da Equação (1).

Dada a fragilidade em estimar a Equação (1) com um modelo MVSPROBIT, faz-se necessário a utilização de um estimador alternativo que controle a endogeneidade da variável inovação $\left(Y_{i}\right)$. O estimador que satisfaz tal condição é o de variáveis instrumentais. Este estimador alternativo, aqui denominado de IV-PROBIT, utiliza na Equação (1) valores preditos de $Y_{i}$ a partir da equação de mínimos quadrados em primeiro estágio, conforme abaixo ${ }^{3}$ :

$$
Y_{i}=X_{1 i} \Pi_{1}+X_{2 i} \Pi_{2}+V_{i}
$$

Onde $V_{i}$ é o termo de erro aleatório e o vetor $X_{2}$ incorpora variáveis explicativas exógenas adicionais ao modelo. A expressão (2) é a equação na forma reduzida para a variável explicativa endógena $\left(Y_{i}=\right.$ intensidade de inovação).

Um ponto a ser esclarecido é sobre a escolha das variáveis instrumentais do vetor $X_{2}$ a serem utilizadas no trabalho. Uma variável instrumental deve

$3 \mathrm{O}$ modelo IV-PROBIT é um estimador eficiente para $\delta \mathrm{T}$, no entanto a mesma característica não é observada para a estimativa dos erros-padrão. Este problema é solucionado mediante a correção dos erros-padrão conforme Murphy e Topel (1985). 
apresentar alguns atributos específicos, sendo o principal deles a exogeneidade. A variável instrumental $\left(w_{i}\right)$ deve ainda apresentar forte poder explicativo da variável endógena $\left(Y_{i}\right)$, e não apresentar qualquer correlação com a variável dependente $\left(y_{i}\right)$ e seu termo de erro.

Esse trabalho utiliza variáveis de restrição à isenção de tarifas para aquisição de insumos importados e restrição a programas de incentivos ao investimento como variáveis instrumentais para inovação. A estratégia de identificação repousa sobre o argumento de que um fato exógeno à decisão da empresa, tal como a imposição de restrições a incentivos concedidos pelas várias esferas de governo. Tal restrição tende a reduzir a capacidade de inovação das firmas, uma vez que as mesmas vêem reduzidas suas capacidades de utilizar insumos intermediários de melhor qualidade ou repor e modernizar seu equipamento de capital.

Por outro lado, o acesso a estes programas de incentivos fiscais tendem a reduzir o custo de capital das empresas, possibilitando que as mesmas poupem parcelas de seus fundos, ou utilizem estes recursos para inversões em atividades de P\&D.

A modernização do equipamento de capital pode contribuir para os dois tipos de inovações discutidos neste artigo. A inovação de processo pode ser gerada pela simples troca de equipamento de capital, ou seja, a implementação de uma nova máquina pode, por si só, gerar uma inovação de processo. Já a inovação de produto pode ser implementada a partir de novo equipamento caso tal inovação tenha sido concebida em forma de protótipo e sua produção, em larga escala, demande novo equipamento de capital. O acesso facilitado à aquisição de insumos intermediários importados traria benefícios similares aos da modernização de equipamento de capital para a atividade inovadora das firmas, uma vez que tais insumos intermediários podem ser intensivos em investimentos em P\&D.

Alguns pontos adicionais a respeito da estratégia de identificação aqui utilizada merecem destaque. O leitor perceberá que a Equação 2 (a regressão auxiliar do estimador IV-PROBIT) é uma função de produção de conhecimento, onde $Y_{i}$ é a variável explicativa endógena esforço inovativo e os vetores $X_{1}$ e $X_{2}$ incluem regressores exógenos. Estes regressores exógenos também incluem variáveis como gastos em $\mathrm{P} \& \mathrm{D}$ por empregado, interação com universidades para desenvolvimento e absorção de inovações, além dos instrumentos já mencionados.

\subsection{Resultados}

Os resultados obtidos da estimativa do modelo econométrico especificado pelas Equações (1) e (2) são reportados na Tabela 2. Foram utilizadas duas 
especificações diferentes para o modelo econométrico: na primeira especificação, denominado modelo 1, as variáveis de restrição (Restrição Incentivo Importação, Restrição Incentivo Federal e Restrição Incentivo Municipal) são utilizadas separadamente como instrumentos.

Tabela 2 - Resultados do modelo econométrico, IV-PROBIT

\begin{tabular}{|c|c|c|c|c|}
\hline \multicolumn{5}{|c|}{ Regressão Segundo Estágio (IV-PROBIT) } \\
\hline \multirow{4}{*}{ Variáveis independentes } & \multirow{3}{*}{\multicolumn{2}{|c|}{$\begin{array}{c}\text { Variável dependente } \\
\text { Internacionalização } \\
\text { (Modelo 1) }\end{array}$}} & \multirow{3}{*}{\multicolumn{2}{|c|}{$\begin{array}{c}\text { Variável dependente } \\
\text { Internacionalização } \\
\text { (Modelo 2) }\end{array}$}} \\
\hline & & & & \\
\hline & & & & \\
\hline & Coeficiente & $\begin{array}{c}\text { Efeitos } \\
\text { Marginais }\end{array}$ & Coeficiente & $\begin{array}{c}\text { Efeitos } \\
\text { Marginais }\end{array}$ \\
\hline \multicolumn{3}{|l|}{ Esforço Inovativo } & $\begin{array}{r}0,91 \\
(0,006)^{* * * *}\end{array}$ & $\begin{array}{r}0,23 \\
(0,001)^{* * * *}\end{array}$ \\
\hline \multicolumn{5}{|c|}{ Regressão Primeiro Estágio, Regressão Auxiliar (IV-PROBIT) } \\
\hline Variáveis independentes & \multicolumn{2}{|c|}{$\begin{array}{l}\text { Variável endógena } \\
\text { Esforço Inovativo }\end{array}$} & \multicolumn{2}{|c|}{$\begin{array}{l}\text { Variável endógena } \\
\text { Esforço Inovativo }\end{array}$} \\
\hline Restrição Incentivo Importação & & $\begin{array}{r}-0,09 \\
(0,020)^{* * * *}\end{array}$ & & \\
\hline Restrição Incentivo Federal & & $\begin{array}{r}0,03 \\
(0,017)^{*}\end{array}$ & & \\
\hline Restrição Incentivo Municipal & & $\begin{array}{r}-0,016 \\
(0,014)\end{array}$ & & \\
\hline Índice de Restrição a Incentivos & & & & $(0,006)^{-0,021}$ \\
\hline Gastos P\&D & & $\begin{array}{r}6,4 \mathrm{E}-06 \\
(1,5 \mathrm{E}-06)^{* * *}\end{array}$ & & $\begin{array}{r}4,3 \mathrm{E}-06 \\
(1,6 \mathrm{E}-06)^{* * *}\end{array}$ \\
\hline Interação Universidade-Empresa & & $\begin{array}{r}0,077 \\
(0,035)^{* *}\end{array}$ & & $\begin{array}{r}0,044 \\
(0,020)^{*}\end{array}$ \\
\hline
\end{tabular}

Fonte: Elaborado pelo próprio autor.

Nota: (1) Erro-padrão entre parênteses; (2) Nível de significância: $\left.{ }^{(* * *}\right)$ para $\left.\alpha=0,01,{ }^{(* *}\right)$ para $\alpha=0,05$, e $\left(^{*}\right)$ para $\alpha=0,10$; (3) Todas as regressões incluem uma constante e demais controles: Escolaridade, Emprego, Idade da Firma, Dummies de Local e Dummies de Setor.

Já na segunda especificação, denominada modelo 2, estas variáveis são utilizadas para a construção de uma única variável instrumental, denominada Índice de Restrição a Incentivos. Este índice foi obtido a partir de técnicas de análise multivariada, mais especificamente, utilizou-se a técnica de análise de componentes principais para atribuir os pesos para cada uma das variáveis que constituíram o índice. Os detalhes da construção do índice são fornecidos no Anexo A.

O objetivo da construção do Índice de Restrição a Incentivos foi o de expressar em uma única variável o aspecto multidimensional de restrições às políticas públicas de incentivos. Cabe observar que a construção deste índice não altera as hipóteses de exogeneidade associadas a cada um dos instrumentos individuais.

Os resultados apresentados na Tabela 2 estão divididos em resultados de primeiro estágio, obtidos na regressão auxiliar (também denominada de função de produção de conhecimento) e de segundo estágio, ou seja, as estimativas do 
coeficiente da variável esforço inovativo. Os coeficientes da regressão de segundo estágio são apresentados no topo da tabela, enquanto que abaixo destes são reportados os resultados da regressão auxiliar. Juntamente com as estimativas do coeficiente da variável esforço inovativo, obtidas por estimadores IV-PROBIT, são reportados seus respectivos efeitos marginais.

Analisando os resultados do modelo 1, contatamos que o coeficiente estimado para a variável esforço inovativo é positivo $(0,90)$ e estatisticamente significativo (nível de significância de $\alpha=0,01$ ). A interpretação do coeficiente do modelo PROBIT não é trivial, de modo que seu efeito marginal também é reportado. $\mathrm{O}$ efeito marginal de $0,23 \mathrm{implica}$ que cada unidade adicional de esforço inovativo equivale a um aumento de 23 pontos percentuais na probabilidade condicional de internacionalização da firma.

Na regressão auxiliar do modelo 1 pode-se constatar que tanto os gastos em $\mathrm{P} \& \mathrm{D}$ quanto a interação com universidades exercem efeitos positivos e significativos sobre o esforço inovativo das empresas. Quando a análise repousa sobre as variáveis de restrições, os efeitos não são tão claros. Espera-se, como já mencionado, que tais variáveis exerçam efeitos negativos sobre o esforço inovativo das empresas. Verifica-se que apenas a variável Restrição Incentivo Importação apresenta sinal coerente e estatisticamente significativo. O coeficiente da variável Restrição Incentivo Federal é estatisticamente significativo, porém apresenta sinal contraintuitivo. Já o coeficiente da variável Restrição Incentivo Municipal apresenta sinal coerente, porém não é significativo.

A alta multicolinearidade destes regressores pode ser fonte de problemas para as estimativas do modelo 1. Com o objetivo de reduzir tal efeito, foi desenvolvido um índice que capturasse toda a informação destes regressores em uma única variável, denominado Índice de Restrição a Incentivos, como já mencionado nos parágrafos anteriores.

Os resultados com este segundo instrumento são reportados na Tabela 2, na coluna denominada Modelo 2. Podemos verificar nos resultados do primeiro estágio da regressão, ou seja, na função de produção de conhecimento, que o coeficiente do instrumento Índice de Restrição a Incentivos apresenta sinal negativo e coerente com a hipótese teórica, a saber, maiores restrições a incentivos fiscais ou governamentais reduzem a capacidade inovativa das firmas. Cabe mencionar ainda que o coeficiente desta variável instrumental é estatisticamente significativo.

Já na equação de segundo estágio (equação de internacionalização) do modelo 2, o coeficiente do modelo probabilístico e seu respectivo efeito marginal são similares àqueles obtidos no modelo 1 , ou seja, novamente constata-se um efeito marginal de 0,23 , o que implica que cada unidade adicional de esforço inovativo equivale a um aumento de 23 pontos percentuais na probabilidade 
condicional de internacionalização da firma, como já mencionado.

\section{Considerações finais}

Este artigo teve como objetivo estimar o efeito da produção de conhecimento tecnológico das empresas industriais brasileiras sobre suas respectivas probabilidades condicionais de internacionalização. Foi discutido ao longo deste artigo que a estimação de tal efeito não é trivial, uma vez que as variáveis esforço de inovação e internacionalização da firma são simultaneamente determinadas. Por conta da especificidade causal entra estas variáveis, optou-se por uma estratégia empírica baseada na utilização de variáveis instrumentais.

A hipótese central deste trabalho é de que empresas inovadoras apresentam maior probabilidade de internacionalização. No entanto, a construção desta hipótese demanda duas outras hipóteses auxiliares: (i) as empresas mais produtivas se auto-selecionam para ingressar no mercado exportador; (ii) o esforço inovativo e a capacidade das firmas em absorver e produzir conhecimento (tanto internamente, quanto para o mercado) estão entre as principais origens da heterogeneidade produtiva das empresas.

Os principais resultados deste trabalho sugerem que, dependendo da capacidade inovativa da empresa (inovadora em processo, inovadora em produto ou inovadora em ambas as atividades), sua probabilidade condicional de ingresso no mercado externo pode aumentar, no mínimo, em 23 pontos percentuais.

Em termos de políticas públicas de promoção de exportações, os resultados deste artigo contribuem para a literatura ao sugerir que instrumentos de disseminação da cultura inovadora podem ser eficientes para o ingresso e permanência de um número cada vez maior de empresas brasileiras no setor exportador.

Finalmente, faz-se necessário mencionar que os resultados apresentados ao longo deste trabalho demandam cautela e as questões aqui endereçadas demandam testes empíricos adicionais, preferencialmente com a utilização de bases de dados alternativas, tais como os microdados da Pesquisa Industrial de Inovação Tecnológica, Pintec/IBGE. A utilização de bases de dados alternativas poderia viabilizar não somente a utilização de outras variáveis instrumentais, bem como de diferentes estratégias empíricas de identificação.

\section{Referências}

Araújo, B. C. P. O.; Kannebley JR., S.; Esteves, L. A. (2009). "Why do industrial firms in developing countries grow after they begin exporting? Evidence from Brazil". Third Conference on Micro Evidence on Innovation and Development, Rio de 
Janeiro.

Araújo, B. C. P. O. (2006). "Potencial das firmas industriais brasileiras e a dimensão tecnológica”. In: DeNegri, J. A.; Araújo, B. C. P. O. (Org). (2006). As empresas brasileiras e o comércio internacional, IPEA: Brasiúlia.

Bernard, A. B.; Jensen, B. (1999). "Exceptional exporter performance: cause, effect, or both?". Journal of International Economics 47: 1-25.

Bernard, A. B.; Jensen, B. (2004). "Why some firms export?". The Review of Economics and Statistics 86: 561-569.

De Negri, J. A., Esteves, L. A.; Freitas, F. (2007). "Knowledge production and firm growth in Brazil". Working Papers 0020, Universidade Federal do Paraná, Department of Economics.

Esteve-Perez, S.; Rodriguez, D. (2009). "The dynamics of trade and innovation: a joint approach”. Mimeo: Universidad Complutense de Madrid.

Girma, S.; Kneller, R. A.; Pisu, M. (2005). "Exports versus FDI: an empirical test". Review of World Economics 141(2): 193-218;

Greenaway, D.; Kneller, R. (2007). "Firm heterogeneity, exporting and foreign direct investment”. Economic Journal 117(517): F134-F161;

Grossman, G. M.; Helpman, E. (1991). Innovation and growth in the global economy. Cambridge, MA: The MIT Press.

Helpman, E., Melitz, M. J.; Yeaple, S. R. (2004). "Export versus FDI with heterogeneous firms". American Economic Review 94(1): 300-316.

Markwald, R.; Puga, F. P. (2002). "Focando a política de promoção às exportações". In: $O$ desafio das exportações, BNDES: Rio de Janeiro.

Melitz, M. J. (2003). "The impact of trade on intra-industry reallocations and aggregate industry productivity”. Econometrica 71: 1695-1725.

Murphy, K. M.; Topel, R. H. (1985). "Estimation and inference in two-step econometric models". Journal of Business and Economic Statistics 3(4), 370-379.

Prochnik, V. Esteves, L. A.; Freitas, F. M. (2006). "O grau de internacionalização das empresas industriais brasileiras”. In: DeNegri, J. A.; Araújo, B. C. P. O. (Org). (2006). As empresas brasileiras e o comércio internacional, IPEA: Brasiília.

Yatchew, A., Griliches, Z. (1985). "Specification error in probit models". The Review of Economics and Statistics 67(1): 134-139.

Wagner, J. (2006). "Exports, foreign direct investment, and productivity". Applied Economics Letters 13(6): 347-349. 
Anexo A

\section{Construção do Índice de Restrição a Incentivos}

A análise de correlação/componentes principais para as variáveis RestriçãoIncentivo Importação, Restrição Incentivo Federale Restrição Incentivo Municipal geraram três componentes principais, com as características abaixo:

Tabela A.1 - Componentes principais

\begin{tabular}{l|c|c|c}
\hline \multicolumn{1}{c|}{ Componente } & Autovalor & Proporção & Acumulada \\
\hline Comp1 & 2,45 & 0,8171 & 0,8171 \\
Comp2 & 0,32 & 0,1075 & 0,9247 \\
Comp3 & 0,22 & 0,0753 & 1,0000 \\
\hline
\end{tabular}

Fonte: Elaborado pelo próprio autor.

O primeiro componente principal, Comp1, é o único componente com autovalor superior à unidade, logo o único a ser utilizado por critério de relevância explicativa da variância. A proporção explicada do Comp1 é de 81,71\%.

Utilizando o método "pcf" (fator de componentes principais) para a obtenção dos pesos do índice, foram obtidos os seguintes resultados:

Tabela A.2 - Pesos

\begin{tabular}{l|c}
\hline \multicolumn{1}{c|}{ Variável } & Fator1 \\
\hline Restrição Incentivo Importação & 0,8853 \\
Restrição Incentivo Federal & 0,9201 \\
Restrição Incentivo Municipal & 0,9062 \\
\hline Fonte: Elaborado pelo próprio autor.
\end{tabular}

O teste de adequação amostral de Kaiser-Meyer-Olkin (KMO) foi conduzido para a eliminação de possíveis variáveis insatisfatórias para os fins de análise de componentes principais. KMO assume valores entre zero e um e valores inferiores a 0,5 são denominados como inaceitáveis e devem ser eliminados da análise. No entanto, nenhuma das variáveis apresentou estatística KMO inferior a 0,70, conforme pode ser visto na tabela abaixo:

Tabela A.3 - Teste KMO

\begin{tabular}{l|c}
\hline \multicolumn{1}{c|}{ Variável } & KMO \\
\hline Restrição Incentivo Importação & 0,7917 \\
Restrição Incentivo Federal & 0,7029 \\
Restrição Incentivo Municipal & 0,7340 \\
\hline \multicolumn{2}{l}{ Fonte: Elaborado pelo próprio autor. }
\end{tabular}

Finalmente, o Índice de Restrição a Incentivos foi construído a partir da seguinte equação:

Índice de Restrição a Incentivos $=0,8853$ (Restrição Incentivo Importação) 
O Índice de Restrição a Incentivos apresentou média de 1,998 e desvio padrão de 1,070, tendo valor mínimo de 0 e máximo de 2,711 . O gráfico da distribuição do índice segue abaixo:

Gráfico 1 - Índice de Restrição a Incentivos

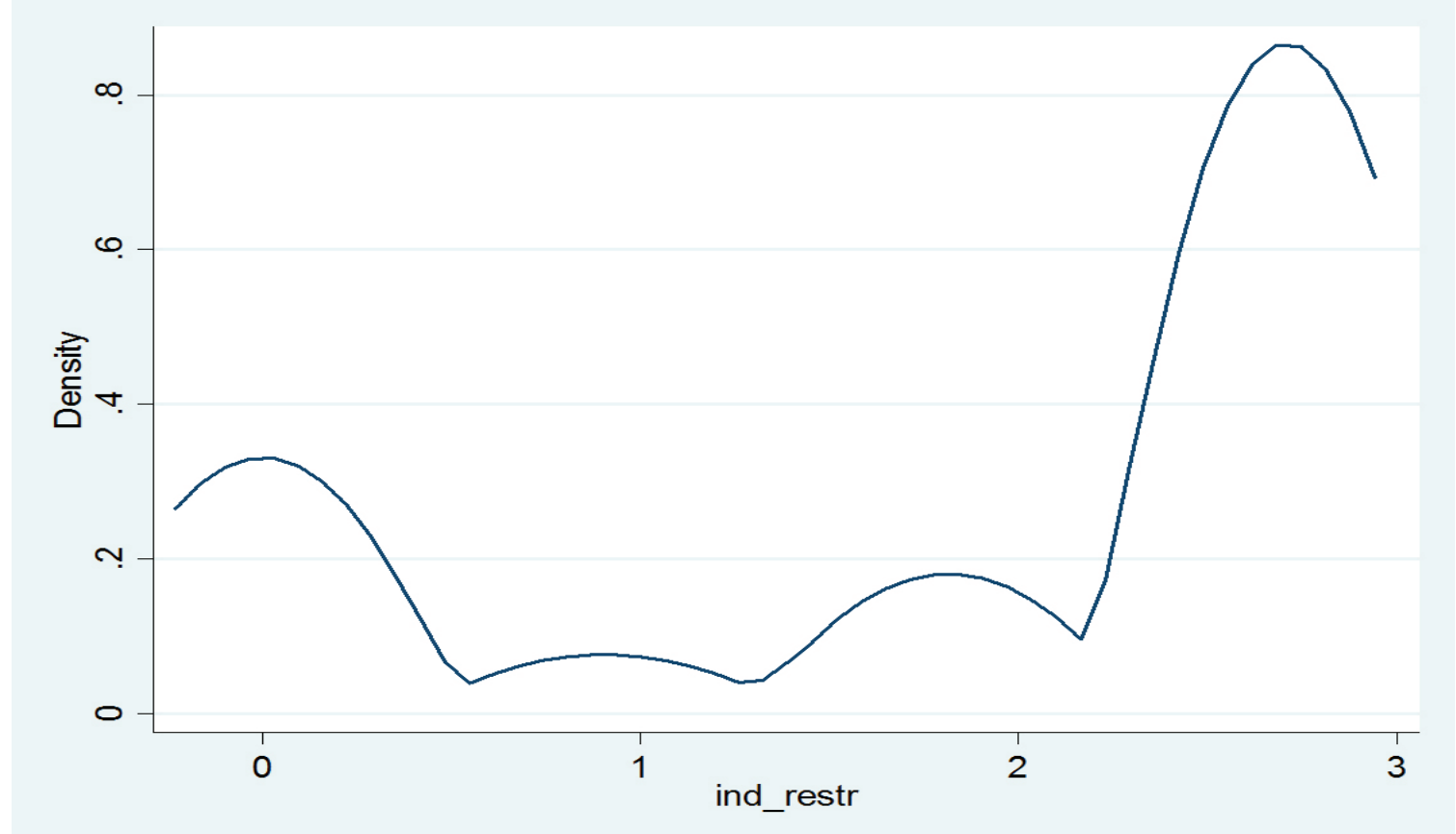

Fonte: Elaborado pelo próprio autor. 
